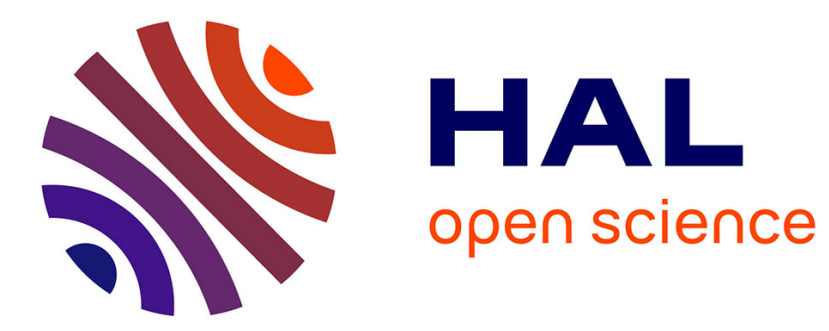

\title{
The Confluence Problem for Flat TRSs
}

Ichiro Mitsuhashi, Michio Oyamaguch, Florent Jacquemard

\section{To cite this version:}

Ichiro Mitsuhashi, Michio Oyamaguch, Florent Jacquemard. The Confluence Problem for Flat TRSs. 8th International Conference Artificial Intelligence and Symbolic Computation (AISC), Sep 2006, Beijing, China. pp.68-81, 10.1007/11856290_8 . inria-00579010

\section{HAL Id: inria-00579010 https://hal.inria.fr/inria-00579010}

Submitted on 23 Mar 2011

HAL is a multi-disciplinary open access archive for the deposit and dissemination of scientific research documents, whether they are published or not. The documents may come from teaching and research institutions in France or abroad, or from public or private research centers.
L'archive ouverte pluridisciplinaire HAL, est destinée au dépôt et à la diffusion de documents scientifiques de niveau recherche, publiés ou non, émanant des établissements d'enseignement et de recherche français ou étrangers, des laboratoires publics ou privés. 


\title{
The Confluence Problem for Flat TRSs
}

\author{
Ichiro Mitsuhashi ${ }^{1}$, Michio Oyamaguch ${ }^{1}$, and Florent Jacquemard ${ }^{2}$ \\ 1 Mie University \\ \{ichiro, mo\}@cs.info.mie-u.ac.jp \\ 2 INRIA-Futurs and LSV, UMR CNRS ENS Cachan \\ florent. jacquemard@lsv. ens-cachan.fr
}

\begin{abstract}
We prove that the properties of reachability, joinability and confluence are undecidable for flat TRSs. Here, a TRS is flat if the heights of the left and right-hand sides of each rewrite rule are at most one.
\end{abstract}

Key words: Term rewriting system, Decision problem, Confluence, Flat.

\section{Introduction}

A term rewriting system (TRS) is a set of directed equations called rewrite rules. It defines a binary relation on terms by replacement of a subterm matching a left member of a rewrite rule by the corresponding right member. A TRS is called confluent (or Church-Rosser) if any two terms obtained, by the rewriting relation, from the same term are joinable. The confluence is a crucial property for the application of rewriting as a model for computation as it ensures the uniqueness of normal forms [1], and it has received much attention so far.

Confluence is undecidable in general, and even for restricted classes of TRS like monadic or semi-constructor TRSs [11]. On the other hand, decidability results have been established for several classes of TRSs, like e.g. ground (rewrite rules having no variables) TRSs [13,4,2], flat (left and right members of rewrite rules having height at most one) and rule-linear (a variable cannot occur more than once in a rewrite rule) TRSs [17], and more recently for flat and rightlinear (a variable cannot occur more than once in a right member of rewrite rule) TRSs [6].

In this paper, we demonstrate that the above linearity restriction is necessary for decidability, showing that confluence is undecidable for flat TRSs, even with only one non-right-linear flat rewrite rule. A previous proof of this result has been published in [8]. However, we have found some technical flaws in this proof. This paper presents a correct and detailed undecidability proof, which is also significantly simpler than the one of [8].

The related properties of reachability (whether a given term can be reached from another given term by rewriting) and joinability (whether two given terms can be rewritten to the same term) are decidable for right-ground (right members of rewrite rules have no variable) TRSs [14], for right-linear monadic TRSs [15, 12], and for right-linear and finite-path-overlapping TRSs [16]. The latter two 
classes properly include the class of flat and right-linear TRSs. We show in this paper that reachability and joinability are undecidable if we drop the rightlinearity condition, i.e. it is undecidable for general flat TRSs.

The paper is organized as follows: after giving the definitions and notations in Section 2, we show in Section 3 that reachability is undecidable for flat TRSs by reduction of the Post's correspondence problem. It follows as a corollary that joinability is also undecidable for the same class. Then, in Section 4, we show that confluence is undecidable for flat TRSs, by a reduction of reachability.

\section{Preliminaries}

We assume that the reader is familiar with the standard definitions of rewrite systems $[5,1]$ and we just recall here the main notations used in this paper.

Let $\varepsilon$ be the empty string. Let $X$ be a set of variables. Let $F$ be a finite set of operation symbols graded by an arity function ar: $F \rightarrow \mathbb{N}(=\{0,1,2, \cdots\})$, $F_{n}=\{f \in F \mid \operatorname{ar}(f)=n\}$. Let $T$ be the set of terms built from $X$ and $F$. A substitution is a finite mapping from $X$ to $T$. As usual, we identify substitutions with their morphism extension to terms, and we use a postfix notation for the application of substitutions. We use $x$ as a variable, $f, h$ as function symbols, $r, s, t$ as terms, $\theta$ as a substitution. A term $s$ is ground if $s$ has no variable. The height of a term is defined as follows: height $(a)=0$ if $a$ is a variable or a constant and height $\left(f\left(t_{1}, \ldots, t_{n}\right)\right)=1+\max \left\{\operatorname{height}\left(t_{1}\right), \ldots\right.$, height $\left.\left(t_{n}\right)\right\}$ if $n>0$.

A position in a term a sequence of positive integers, and positions are partially ordered by the prefix ordering $\geq$. Let $\left.s\right|_{p}$ be the subterm of $s$ at position $p$. Let $s \geq_{\text {sub }} t$ if $t$ is a subterm of $s$. For a position $p$ and a term $t$, we use $s[t]_{p}$ to denote the term obtained from $s$ by replacing the subterm $\left.s\right|_{p}$ by $t$.

A rewrite rule $\alpha \rightarrow \beta$ is a directed equation over terms. A TRS $R$ is a finite set of rewrite rules. A term $s$ reduces to $t$ at position $p$ by the TRS $R$, denoted $s \frac{p}{R} t$ ( $p$ and $R$ may be omitted), if $\left.s\right|_{p}=\alpha \theta$ and $t=s[\beta \theta]_{p}$ for some rewrite rule $\alpha \rightarrow \beta$ and substitution $\theta$. Let $\stackrel{=}{\longrightarrow}$ be $\rightarrow \cup=\leftarrow$ be the inverse of $\rightarrow$ and $\stackrel{*}{\rightarrow}$ be the reflexive and transitive closure of $\rightarrow$. The terms $s$ and $t$ are joinable if $s \stackrel{*}{\longrightarrow} \cdot \stackrel{*}{\longleftarrow}$, which is denoted $s \downarrow t$. The term $t$ is reachable from $s$ if $s \stackrel{*}{\longrightarrow} t$. The term $r$ is confluent on the TRS $R$ if for every peak $s \stackrel{*}{R} r \stackrel{*}{R} t$, we have $s \downarrow t$. The TRS $R$ is confluent if every term is confluent on $R$. Let $\gamma: s_{1} \stackrel{p_{1}}{\longrightarrow} s_{2} \ldots \stackrel{p_{n-1}}{\longrightarrow} s_{n}$ be a rewrite sequence. This sequence is abbreviated by $\gamma: s_{1} \stackrel{*}{\longrightarrow} s_{n}$ and $\gamma$ is called $p$-invariant if $p_{i}>p$ for every redex position $p_{i}$ of $\gamma$; this is denoted by $\gamma: s_{1} \stackrel{>p *}{\longrightarrow} s_{n}$.

Definition 1. A rule $\alpha \rightarrow \beta$ is flat if height $(\alpha) \leq 1$ and height $(\beta) \leq 1$. A rule $\alpha \rightarrow \beta$ is monadic if height $(\beta) \leq 1$. A term $s$ is shallow if $s$ is a variable or $s=f\left(s_{1}, \cdots, s_{n}\right)$ for some function symbol $f$ and terms $s_{1}, \cdots, s_{n}$ such that every $s_{i}(1 \leq i \leq n)$ is either a variable or ground. A rule $\alpha \rightarrow \beta$ is shallow if both $\alpha$ and $\beta$ are shallow. For $\mathcal{C} \in$ \{flat, monadic, shallow $\}, R$ is $\mathcal{C}$ if every rule in $R$ is $\mathcal{C}$.

We are interested in the following decision problems: 
Reachability Given a TRS $R$ and two terms $s, t$, does there exist a rewrite sequence $s \stackrel{*}{R} t$ ?

Joinability Given a TRS $R$ and two terms $s, t$, are $s$ and $t$ joinable, i.e., $s \downarrow_{R} t$ ?

Confluence Given a TRS $R$, is every term confluent on $R$ ?

Definition 2. A finite automaton is a 5 -tuple $\left(Q, \Sigma, \delta, F_{Q}, q_{0}\right)$ where $Q$ is a finite set of states, $\Sigma$ is a finite set of input symbols, $\delta: Q \times \Sigma \rightarrow Q$ is a function, $F_{Q} \subseteq Q$ is a finite set of final states, and $q_{0} \in Q$ is the initial state.

\section{Reachability and joinability for flat TRSs}

In [8], it has been reported that reachability and joinability are also undecidable for flat TRSs. But, the undecidability proof of reachability contains a flaw. We propose a repaired proof of undecidability for reachability which is simpler than the former one. The proof is a reduction of the Post's Correspondence Problem (PCP) into the reachability of a constant 1 from a constant 0 using a flat TRS $R_{1}$. This TRS, constructed from the given instance of PCP, is such that every rewrite sequence $0 \stackrel{*}{\stackrel{*}{R_{1}}} 1$ contains a representation of a solution of the PCP as a term $t$. This property is ensured, informally, by running separately several sub-TRS of $R_{1}$ on several copies of $t$, where all copies have different colors and are under a function symbol of arity 6 or 7 . Moreover, some equality tests are performed during the rewrite sequence using $R_{1}$, by means of a flat rewrite rules in $R_{1}$ containing some non-linear variables.

Let $P=\left\{\left\langle u_{i}, v_{i}\right\rangle \in \Sigma^{+} \times \Sigma^{+} \mid 1 \leq i \leq k\right\}$ be an instance of PCP. The goal of the problem is to find a sequence of indices $i_{1}, \ldots, i_{n}$, possibly with repetitions, such that the concatenations $u_{i_{1}} \ldots, u_{i_{n}}$ and $v_{i_{1}} \ldots, v_{i_{n}}$ are equal. Note that the alphabet $\Sigma$ is fixed. Let $l_{P}=\max _{1 \leq i \leq k}\left(\left|u_{i}\right|,\left|v_{i}\right|\right)$. Let $\_$be a new symbol and $\Delta=\left\{1, \cdots, l_{P}\right\} \times\left(\Sigma \cup\left\{{ }_{-}\right\}\right)^{2}$. We shall use a product operator $\otimes$ which associates to two non-empty strings of $\Sigma^{+}$of length smaller than or equal to $l_{P}$ a word of $\Delta^{*}$ of length $l_{P}$ as follows: $a_{1} \cdots a_{n} \otimes a_{1}^{\prime} \cdots a_{m}^{\prime}=\left\langle 1, a_{1}, a_{1}^{\prime}\right\rangle \cdots\left\langle l_{P}, a_{l_{P}}, a_{l_{P}}^{\prime}\right\rangle$, where $a_{1}, \cdots, a_{n}, a_{1}^{\prime}, \cdots, a_{m}^{\prime} \in \Sigma, a_{i}=_{-}$for all $i \in\left\{n+1, \cdots, l_{P}\right\}$, and $a_{j}^{\prime}=_{-}$ for all $j \in\left\{m+1, \cdots, l_{P}\right\}$. Note that $\left\langle 1,_{-},{ }_{-}\right\rangle(s),\left\langle 1,{ }_{-}, a_{1}^{\prime}\right\rangle(s)$, or $\left\langle 1, a_{1},{ }_{-}\right\rangle(s)$ can not be returned by operator $\otimes$.

Example 1. Let $l_{P}=4$, then $\mathrm{a} \otimes \mathrm{bab}=\langle 1, \mathrm{a}, \mathrm{b}\rangle\left\langle 2,_{-}, \mathrm{a}\right\rangle\left\langle 3,_{-}, \mathrm{b}\right\rangle\left\langle 4,_{-},{ }_{-}\right\rangle$.

Let $A=\left(Q_{A}, \Delta, \delta_{A}, F_{Q_{A}}, q_{A}\right)$ and $B=\left(Q_{B}, \Sigma, \delta_{B}, F_{Q_{B}}, q_{B}\right)$ be two finite automata recognizing the respective sets $L(A)=\left\{u_{i} \otimes v_{i} \mid\left\langle u_{i}, v_{i}\right\rangle \in P\right\}^{+}$and $L(B)=\Sigma^{+}$. We may assume that both $q_{A}$ and $q_{B}$ are non final. We assume that the automata $A$ and $B$ are clean (i.e., any state accepts some input string and is reachable from the initial state $q_{A}$ (or $q_{B}$ ) by some input string). We associate automata $A, B$ with the following ground TRSs $T_{A}, T_{B}$, respectively:

$$
\begin{aligned}
& T_{A}^{(i, j)}=\left\{q^{(i)} \rightarrow d^{(j)}\left(q^{(i)}\right) \mid q^{\prime} \in \delta_{A}(q, d)\right\} \cup\left\{q^{(i)} \rightarrow \mathrm{e} \mid q \in F_{Q_{A}}\right\} \\
& T_{B}^{(i, j)}=\left\{q^{(i)} \rightarrow a^{(j)}\left(q^{\prime(i)}\right) \mid q^{\prime} \in \delta_{B}(q, a)\right\} \cup\left\{q^{(i)} \rightarrow \mathrm{e} \mid q \in F_{Q_{B}}\right\}
\end{aligned}
$$


We assume given 13 disjoint copies of the above signatures, colored with color $i \in\{0, \cdots, 12\}$ :

$$
\begin{array}{ll}
\Sigma^{(i)}=\left\{a^{(i)} \mid a \in \Sigma\right\} & Q_{A}^{(i)}=\left\{q^{(i)} \mid q \in Q_{A}\right\} \\
\Delta^{(i)}=\left\{d^{(i)} \mid d \in \Delta\right\} & Q_{B}^{(i)}=\left\{q^{(i)} \mid q \in Q_{B}\right\}
\end{array}
$$

Let $\Theta^{012}=\Delta^{(0)} \cup \Sigma^{(1)} \cup \Sigma^{(2)}, \Theta^{345}=\Delta^{(3)} \cup \Delta^{(4)} \cup \Sigma^{(5)}$, and $Q=Q_{A}^{(6)} \cup Q_{A}^{(7)} \cup$ $Q_{A}^{(8)} \cup Q_{A}^{(9)} \cup Q_{B}^{(10)} \cup Q_{B}^{(11)} \cup Q_{A}^{(12)}$. Let e be a constant. We assume that $\operatorname{ar}(f)=1$ for every $f \in \Delta \cup \Sigma$. For a ground term $t$ built from $\Delta \cup \Sigma \cup\{\mathrm{e}\}, t^{(i)}$ is defined as follows: $\mathrm{e}^{(i)}=\mathrm{e}$ and $\left(f\left(t_{1}\right)\right)^{(i)}=f^{(i)}\left(t_{1}^{(i)}\right)$ for $f \in \Delta \cup \Sigma$ and term $t_{1}$.

Now, we define the flat TRS $R_{1}$ on an extended signature $F_{0}=Q \cup\{\mathrm{e}, 0,1\}$, $F_{1}=\Theta^{012} \cup \Theta^{345}, F_{6}=\{\mathrm{f}\}$, and $F_{7}=\{\mathrm{g}\}$. First, we color $T_{A}$ and $T_{B}$ :

$$
\begin{aligned}
& T_{A}^{(i, j)}=\left\{q^{(i)} \rightarrow d^{(j)}\left(q^{(i)}\right) \mid q^{\prime} \in \delta_{A}(q, d)\right\} \cup\left\{q^{(i)} \rightarrow \mathrm{e} \mid q \in F_{Q_{A}}\right\} \\
& T_{B}^{(i, j)}=\left\{q^{(i)} \rightarrow a^{(j)}\left(q^{\prime(i)}\right) \mid q^{\prime} \in \delta_{B}(q, a)\right\} \cup\left\{q^{(i)} \rightarrow \mathrm{e} \mid q \in F_{Q_{B}}\right\}
\end{aligned}
$$

Next, we define recoloring TRSs $S, P$ and projection TRSs $\Pi_{1}, \Pi_{2}$ :

$$
\begin{aligned}
S^{(i, j)}= & \left\{a^{(i)}(x) \rightarrow a^{(j)}(x) \mid a \in \Sigma\right\} \\
P^{(i, j)}= & \left\{d^{(i)}(x) \rightarrow d^{(j)}(x) \mid d \in \Delta\right\} \\
\Pi_{1}^{(i, j)}= & \left\{\left\langle n, a, a^{\prime}\right\rangle^{(i)}(x) \rightarrow a^{(j)}(x) \mid n \in\left\{1, \cdots, l_{P}\right\}, a \in \Sigma, a^{\prime} \in \Sigma \cup\left\{{ }_{-}\right\}\right\} \\
& \cup\left\{\left\langle n,{ }_{-}, a^{\prime}\right\rangle^{(i)}(x) \rightarrow x \mid n \in\left\{2, \cdots, l_{P}\right\}, a^{\prime} \in \Sigma \cup\left\{{ }_{-}\right\}\right\} \\
\Pi_{2}^{(i, j)}= & \left\{\left\langle n, a, a^{\prime}\right\rangle^{(i)}(x) \rightarrow a^{\prime(j)}(x) \mid n \in\left\{1, \cdots, l_{P}\right\}, a \in \Sigma \cup\left\{\left\{_{-}\right\}, a^{\prime} \in \Sigma\right\}\right. \\
& \cup\left\{\left\langle n, a,{ }_{-}\right\rangle^{(i)}(x) \rightarrow x \mid n \in\left\{2, \cdots, l_{P}\right\}, a \in \Sigma \cup\left\{{ }_{-}\right\}\right\}
\end{aligned}
$$

The flat TRS $R_{1}$ is defined as follows:

$$
\begin{aligned}
& R_{0}= T_{A}^{(6,3)} \cup T_{A}^{(7,3)} \cup T_{A}^{(8,4)} \cup T_{A}^{(9,4)} \cup T_{B}^{(10,5)} \cup T_{B}^{(11,5)} \\
& \cup P^{(3,0)} \cup \Pi_{1}^{(3,1)} \cup \Pi_{2}^{(4,2)} \cup P^{(4,0)} \cup S^{(5,1)} \cup S^{(5,2)} \cup T_{A}^{(12,0)} \\
& R_{1}=R_{0} \cup\left\{\begin{array}{l}
0 \rightarrow \mathrm{f}\left(q_{A}^{(6)}, q_{A}^{(7)}, q_{A}^{(8)}, q_{A}^{(9)}, q_{B}^{(10)}, q_{B}^{(11)}\right), \\
\mathrm{f}\left(x_{3}, x_{3}, x_{4}, x_{4}, x_{5}, x_{5}\right) \rightarrow \mathrm{g}\left(x_{3}, x_{3}, x_{4}, x_{4}, x_{5}, x_{5}, q_{A}^{(12)}\right), \\
\mathrm{g}\left(x_{0}, x_{1}, x_{2}, x_{0}, x_{1}, x_{2}, x_{0}\right) \rightarrow 1
\end{array}\right\}
\end{aligned}
$$


By construction of $R_{1}$, if $0 \stackrel{*}{R_{1}} 1$ then the rules of $R_{1}$ are applied as described in the following picture.

$$
\begin{aligned}
& 0 \stackrel{\varepsilon}{\rightarrow} \mathrm{f}\left(q_{A}^{(6)}, \quad q_{A}^{(7)}, \quad q_{A}^{(8)}, \quad q_{A}^{(9)}, \quad q_{B}^{(10)}, \quad q_{B}^{(11)}\right) \\
& { }^{*} \downarrow_{T_{A}^{(6,3)}} \quad{ }^{*} \downarrow_{T_{A}^{(7,3)}} \quad{ }^{*} \downarrow_{T_{A}^{(8,4)}} \quad{ }^{*} \downarrow_{T_{A}^{(9,4)}} \quad{ }^{*} \downarrow_{T_{B}^{(10,5)}} \quad{ }^{*} \downarrow_{T_{B}^{(11,5)}} \\
& \mathrm{f}\left(t_{3}, \quad t_{3}, \quad t_{4}, \quad t_{4}, \quad t_{5}, \quad t_{5}\right) \\
& \varepsilon \downarrow \\
& \mathrm{g}\left(t_{3}, \quad t_{3}, \quad t_{4}, \quad t_{4}, \quad t_{5}, \quad t_{5}, \quad q_{A}^{(12)}\right) \\
& { }^{*} \downarrow_{P^{(3,0)}} \quad{ }^{*} \downarrow_{\Pi_{1}^{(3,1)}} \quad{ }^{*} \downarrow_{\Pi_{2}^{(4,2)}} \quad{ }^{*} \downarrow_{P^{(4,0)}} \quad{ }^{*} \downarrow_{S^{(5,1)}} \quad{ }^{*} \downarrow_{S^{(5,2)}} \quad{ }^{*} \downarrow_{T_{A}^{(12,0)}} \\
& \mathrm{g}\left(t_{0}, \quad t_{1}, \quad t_{2}, \quad t_{0}, \quad t_{1}, \quad t_{2}, \quad t_{0}\right) \quad \stackrel{\varepsilon}{\rightarrow} 1
\end{aligned}
$$

Indeed, each of the symbols 0 and 1 occurs in only one rewrite rule of $R_{1}$, and there is only one rule to transform the function symbol $\mathrm{f}$ into $\mathrm{g}$.

Moreover, in the above rewrite sequence by $R_{1}$, we have a subsequence $q_{A}^{(12)} \frac{*}{T_{A}^{(12,0)}} t_{0}$, which means that $t_{0}$ has the form $\left(\left(u_{i_{1}} \otimes v_{i_{1}}\right) \cdots\left(u_{i_{m}} \otimes v_{i_{m}}\right)(\mathrm{e})\right)^{(0)}$ for some $i_{1}, \cdots, i_{m} \in\{1, \cdots, k\}$. We will show in Lemma 1 that:

$$
q_{A}^{(6)} \frac{*}{T_{A}^{(6,3)} \cup P^{(3,0)}} t_{0} \stackrel{*}{\stackrel{*}{T_{A}^{(9,4)} \cup P^{(4,0)}}} q_{A}^{(9)}
$$

Figure 1 shows how colors are changed by the rules of $R_{1}$.

Let $G_{1}$ be the set of ground terms built from $F_{0} \cup F_{1} \cup F_{6} \cup F_{7}$.

\section{Definition 3.}

(1) Let $\underset{R}{\stackrel{*}{R}}(s)=\{t \mid s \stackrel{*}{R} t\}$. For a subset $C \subseteq\{0, \cdots, 12\}$, let $G^{C}$ be the intersection of $G_{1}$ and the set of ground terms built from e and colored function symbols in $\cup_{i \in C}\left(\Sigma^{(i)} \cup \Delta^{(i)} \cup Q_{A}^{(i)} \cup Q_{B}^{(i)}\right)$.

(2) The index of an $i$-colored term built from $\Delta^{(i)} \cup\{\mathrm{e}\}$ is a string of integers defined as follows: index $(\mathrm{e})=\varepsilon$, and $\operatorname{index}\left(\left\langle n, a, a^{\prime}\right\rangle^{(i)}(t)\right)=n \operatorname{index}(t)$.

This Lemma 1 will be useful in the proof of the following Lemma 2.

Lemma 1. Assume that $q_{A}^{(6)} \underset{R_{0}}{\longrightarrow} t_{0}$ and $q_{A}^{(9)} \underset{R_{0}}{\stackrel{*}{\longrightarrow}} t_{0}$ where $t_{0}=\left(\left(u_{i_{1}} \otimes v_{i_{1}}\right) \cdots\left(u_{i_{m}} \otimes v_{i_{m}}\right)(\mathrm{e})\right)^{(0)}$. Then, the following properties hold:

(1) $q_{A}^{(6)} \frac{*}{T_{A}^{(6,3)} \cup P^{(3,0)}} t_{0}$

(2) $q_{A}^{(9)} \frac{*}{T_{A}^{(9,4)} \cup P^{(4,0)}} t_{0}$

\section{Proof}




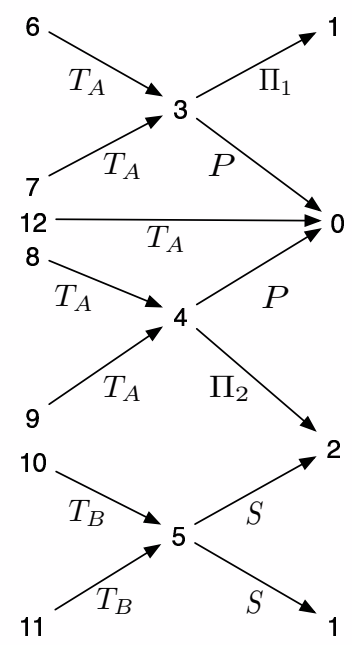

Fig. 1. Graph of the reduction of colors for $R_{1}$.

(1) By definition of $R_{0}, q_{A}^{(6)} \frac{*}{T_{A}^{(6,3)} \cup P^{(3,0)} \cup \Xi_{1}^{(3,1)}} t_{0}$ where $\Xi_{1}^{(3,1)}$ is the subset of $\Pi_{1}^{(3,1)}$ defined by:

$$
\Xi_{1}^{(3,1)}=\left\{\left\langle n,_{-}, a^{\prime}\right\rangle^{(3)}(x) \rightarrow x \mid n \in\left\{2, \cdots, l_{P}\right\}, a^{\prime} \in \Sigma \cup\left\{{ }_{-}\right\}\right\} .
$$

Note that index $\left(t_{0}\right)=\left(1 \cdots l_{P}\right)^{m}$. In this rewrite sequence, if there is at least one application of some rule of $\Xi_{1}^{(3,1)}$, index $\left(t_{0}\right)=\left(1 \cdots l_{P}\right)^{m}$ does not hold, since if we applied a rule $\left\langle n,{ }_{-}, a^{\prime}\right\rangle^{(3)}(x) \rightarrow x$, then at most $m-1$ symbols of $n$ would be included in index $\left(t_{0}\right)$ whereas exactly $m$ symbols of 1 in index $\left(t_{0}\right)$ (since any symbol of form $\left\langle 1, a, a^{\prime}\right\rangle^{(3)}$ can not be deleted). Thus, the proposition holds.

(2) Similar to (1).

Lemma 2. $0 \stackrel{*}{\underset{R_{1}}{\longrightarrow}} 1$ iff the PCP $P$ has a solution.

\section{Proof}

Only if part: by definition of $R_{1}$, we have:

$$
\begin{aligned}
& 0 \underset{R_{1}}{\longrightarrow} \mathrm{f}\left(q_{A}^{(6)}, q_{A}^{(7)}, q_{A}^{(8)}, q_{A}^{(9)}, q_{B}^{(10)}, q_{B}^{(11)}\right) \stackrel{>\varepsilon *}{\stackrel{R_{0}}{\longrightarrow}} \mathrm{f}\left(t_{3}, t_{3}, t_{4}, t_{4}, t_{5}, t_{5}\right)
\end{aligned}
$$

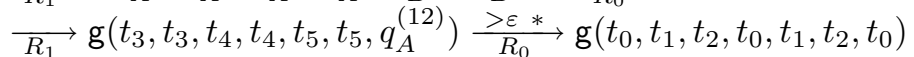

$$
\begin{aligned}
& \underset{R_{1}}{\longrightarrow} 1
\end{aligned}
$$

By definition of $R_{0}$ : 


$$
\begin{aligned}
& \stackrel{*}{\underset{R_{0}}{\longrightarrow}}\left(q_{A}^{(6)}\right) \subseteq G^{\{0,1,3,6\}}, \stackrel{*}{\underset{R_{0}}{\longrightarrow}}\left(q_{A}^{(7)}\right) \subseteq G^{\{0,1,3,7\}}, \\
& \stackrel{*}{\underset{R_{0}}{\longrightarrow}}\left(q_{A}^{(8)}\right) \subseteq G^{\{0,2,4,8\}}, \stackrel{*}{\underset{R_{0}}{\longrightarrow}}\left(q_{A}^{(9)}\right) \subseteq G^{\{0,2,4,9\}}, \\
& \stackrel{*}{\stackrel{*}{R_{0}}}\left(q_{B}^{(10)}\right) \subseteq G^{\{1,2,5,10\}}, \underset{R_{0}}{\stackrel{*}{\longrightarrow}}\left(q_{B}^{(11)}\right) \subseteq G^{\{1,2,5,11\}}
\end{aligned}
$$

We first show that the following condition (I) holds:

$$
t_{i} \in G^{\{i\}} \quad \forall i \in\{0, \cdots, 5\}
$$

Note that $t_{3} \in G^{\{0,1,3\}}$ holds, since $t_{3} \in \underset{R_{0}}{\stackrel{*}{\longrightarrow}}\left(q_{A}^{(6)}\right) \cap \underset{R_{0}}{\stackrel{*}{\longrightarrow}}\left(q_{A}^{(7)}\right)$.

Similarly, $t_{4} \in G^{\{0,2,4\}}$ and $t_{5} \in G^{\{1,2,5\}}$.

Since $t_{3} \underset{R_{0}}{\stackrel{*}{\longrightarrow}} t_{0}$ and $t_{4} \underset{R_{0}}{\stackrel{*}{\longrightarrow}} t_{0}$, we have $t_{0} \in G^{\{0,1,3\}} \cap G^{\{0,2,4\}}=G^{\{0\}}$.

Similarly, $t_{1} \in G^{\{0,1,3\}} \cap G^{\{1,2,5\}}=G^{\{1\}}$ and $t_{2} \in G^{\{0,2,4\}} \cap G^{\{1,2,5\}}=G^{\{2\}}$.

Hence, the condition (I) holds for $i \in\{0,1,2\}$.

Since $t_{3} \underset{R_{0}}{\stackrel{*}{\longrightarrow}} t_{0} \in G^{\{0\}}$ and $t_{3} \underset{R_{0}}{\stackrel{*}{\longrightarrow}} t_{1} \in G^{\{1\}}, t_{3}$ can not contain any symbol in $G^{\{0,1\}}$, hence $t_{3} \in G^{\{3\}}$ holds.

Similarly, $t_{4} \in G^{\{4\}}$ holds because $t_{4} \underset{R_{0}}{\stackrel{*}{\longrightarrow}} t_{2} \in G^{\{2\}}$ and $t_{4} \underset{R_{0}}{\stackrel{*}{\longrightarrow}} t_{0} \in G^{\{0\}}$, and $t_{5} \in G^{\{5\}}$ holds because $t_{5} \underset{R_{0}}{\stackrel{*}{\longrightarrow}} t_{1} \in G^{\{1\}}$ and $t_{5} \underset{R_{0}}{\stackrel{*}{\longrightarrow}} t_{2} \in G^{\{2\}}$.

Hence, (I) holds for $i \in\{3,4,5\}$, as claimed.

By (I), we have: $t_{3} \underset{\Pi_{1}^{(3,1)}}{\stackrel{*}{\longrightarrow}} t_{1} \underset{S^{(5,1)}}{\stackrel{*}{*}} t_{5}$ and $t_{4} \underset{\Pi_{2}^{(4,2)}}{\stackrel{*}{\longrightarrow}} t_{2} \underset{S^{(5,2)}}{\stackrel{*}{(0)}} t_{5}$.

Since $q_{A}^{(12)} \underset{R_{0}}{\stackrel{*}{\longrightarrow}} t_{0}, t_{0}=\left(\left(u_{i_{1}} \otimes v_{i_{1}}\right) \cdots\left(u_{i_{m}} \otimes v_{i_{m}}\right)(\mathrm{e})\right)^{(0)}$ for some $i_{1}, \cdots, i_{m} \in$ $\{1, \cdots, k\}$. We have $m>0$ because the initial state $q_{A}$ is not final.

By Lemma 1, $t_{3} \underset{P^{(3,0)}}{\stackrel{*}{\longrightarrow}} t_{0} \underset{P^{(4,0)}}{\stackrel{*}{~}} t_{4}$. Thus, $t_{3}=\left(\left(u_{i_{1}} \otimes v_{i_{1}}\right) \cdots\left(u_{i_{m}} \otimes v_{i_{m}}\right)(\mathrm{e})\right)^{(3)}$ and $t_{4}=\left(\left(u_{i_{1}} \otimes v_{i_{1}}\right) \cdots\left(u_{i_{m}} \otimes v_{i_{m}}\right)(\mathrm{e})\right)^{(4)}$.

Since $t_{3} \underset{\Pi_{1}^{(3,1)}}{\stackrel{*}{\longrightarrow}} t_{1}, t_{1}=\left(u_{i_{1}} \cdots u_{i_{m}}(\mathrm{e})\right)^{(1)}$,

and since $t_{4} \underset{\Pi_{2}^{(4,2)}}{\stackrel{*}{\longrightarrow}} t_{2}, t_{2}=\left(v_{i_{1}} \cdots v_{i_{m}}(\mathrm{e})\right)^{(2)}$.

Finally, $t_{1} \underset{S^{(5,1)}}{\stackrel{*}{\longrightarrow}} t_{5} \underset{S^{(5,2)}}{\stackrel{*}{\longrightarrow}} t_{2}$, hence $t_{5}=\left(u_{i_{1}} \cdots u_{i_{m}}(\mathrm{e})\right)^{(5)}=\left(v_{i_{1}} \cdots v_{i_{m}}(\mathrm{e})\right)^{(5)}$. It means that the PCP $P$ has a solution.

If part: let $i_{1} \cdots i_{m}$ be a solution of the PCP $P$, and let:

$$
s=\left(u_{i_{1}} \otimes v_{i_{1}}\right) \cdots\left(u_{i_{m}} \otimes v_{i_{m}}\right)(\mathrm{e}) \text { and } t=u_{i_{1}} \cdots u_{i_{m}}(\mathrm{e})
$$

Then, $t=v_{i_{1}} \cdots v_{i_{m}}(\mathrm{e})$ holds. By definition of $R_{1}$, we have:

$$
\begin{aligned}
0 & \rightarrow \mathrm{f}\left(q_{A}^{(6)}, q_{A}^{(7)}, q_{A}^{(8)}, q_{A}^{(9)}, q_{B}^{(10)}, q_{B}^{(11)}\right) \stackrel{*}{\longrightarrow} \mathrm{f}\left(s^{(3)}, s^{(3)}, s^{(4)}, s^{(4)}, t^{(5)}, t^{(5)}\right) \\
& \rightarrow \mathrm{g}\left(s^{(3)}, s^{(3)}, s^{(4)}, s^{(4)}, t^{(5)}, t^{(5)}, q_{A}^{(12)}\right) \stackrel{*}{\longrightarrow} \mathrm{g}\left(s^{(0)}, t^{(1)}, t^{(2)}, s^{(0)}, t^{(1)}, t^{(2)}, s^{(0)}\right) \\
& \rightarrow 1
\end{aligned}
$$

Hence, $0 \underset{R_{1}}{\stackrel{*}{\longrightarrow}} 1$

As a consequence of Lemma 2, we have the following main theorem of this section. 
Theorem 1. Reachability is undecidable for flat TRSs.

Since 1 is a normal form, $0 \stackrel{*}{R_{1}} 1$ iff $0 \downarrow_{R_{1}}$. Thus, the following corollary holds.

Corollary 1. Joinability is undecidable for flat TRSs.

Compared to the construction in [8] for Lemma 2, on the one hand, the above TRS $R_{1}$ is simpler and on the other hand, some rules have been added in order to permit the reduction $q_{A}^{(12)} \frac{*}{T_{A}^{(12,0)}} t_{0}$. This appeared to be necessary in order to fix a bug [8] where a reduction $0 \stackrel{*}{\longrightarrow} 1$ was possible with the TRS associated to the PCP $\{\langle a a, a\rangle,\langle a, a b\rangle\}$ whereas it has no solution. The main reason for this counter-example is for lack of some consideration such as Lemma 1 above (derived from the existence of $q_{A}^{(12)} \stackrel{*}{\longrightarrow}\left(\left(u_{i_{1}} \otimes v_{i_{1}}\right) \cdots\left(u_{i_{m}} \otimes v_{i_{m}}\right)(\mathrm{e})\right)^{(0)}$ and the definition of operator $\otimes$ ).

\section{Confluence for flat TRSs}

We show that confluence for flat TRSs is undecidable by reduction of the reachability problem which has been shown undecidable in the previous section. We introduce some technical definitions in Sections 4.1.

\subsection{Mapping lemma}

A mapping $\phi$ from $T$ to $T$ can be extended to TRSs as follows:

$$
\phi(R)=\{\phi(\alpha) \rightarrow \phi(\beta) \mid \alpha \rightarrow \beta \in R\} \backslash\{t \rightarrow t \mid t \in T\}
$$

Such a mapping $\phi$ can also be extended to substitutions by $\phi(\theta)=\{x \mapsto \phi(x \theta) \mid$ $x$ in the domain of $\theta$ \}. The following lemma gives a characterization of confluence for a TRS $R$ using $\phi(R)$.

Lemma 3. A TRS $R$ is confluent iff there exists a mapping $\phi: T \rightarrow T$ that satisfies the following conditions (1)-(4).

(1) If $s \underset{R}{\longrightarrow} t$ then $\phi(s) \underset{\phi(R)}{\stackrel{*}{\longrightarrow}} \phi(t)$

(2) $\underset{\phi(R)}{\stackrel{\subseteq}{R}} \stackrel{*}{\longrightarrow}$

(3) $t \stackrel{*}{R} \phi(t)$

(4) $\phi(R)$ is confluent

Proof Only if part: let $\phi$ be the identity mapping.

If part: assume that $s \stackrel{*}{\stackrel{*}{R}} r \underset{*}{\stackrel{*}{R}} t$.

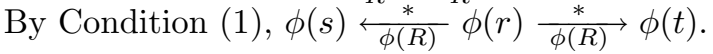

By Condition (4), $\phi(s) \downarrow_{\phi(R)} \phi(t)$.

By Condition (2), $\phi(s) \downarrow_{R} \phi(t)$.

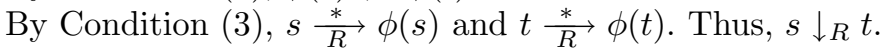

This lemma is used in Section 4.2. 


\subsection{Proof of undecidability}

Let us introduce new function symbols $\Theta_{2}^{012}=\left\{d_{2} \mid d \in \Theta^{012}\right\}$, where each $d_{2}$ has arity 2 . We add the following rules to the TRS $R_{1}$ of Section 3:

$$
R_{2}=R_{1} \cup\{\mathrm{e} \rightarrow 0\} \cup\left\{d(x) \rightarrow d_{2}(0, x), d_{2}(1, x) \rightarrow x \mid d \in \Theta^{012}\right\}
$$

Note that the TRS $R_{2}$ is flat. Let $G_{2}$ be the set of ground terms built from $F_{0} \cup F_{1} \cup F_{6} \cup F_{7} \cup \Theta_{2}^{012}$.

First, we show that $0 \underset{R_{2}}{\stackrel{*}{\longrightarrow}} 1$ iff $0 \underset{R_{1}}{\stackrel{*}{\longrightarrow}} 1$. For this purpose, we will introduce another reduction mapping $\phi$ and another TRS $R_{2}^{\prime}$ and show a technical lemma.

Let $\psi$ be the mapping over $G_{2}$ defined as follows.

$$
\begin{aligned}
\psi\left(h\left(t_{1}, \cdots, t_{n}\right)\right) & =\mathrm{e} & & \text { (if } h \in\{0,1, \mathrm{f}, \mathrm{g}\}) \\
\psi\left(d_{2}\left(t_{1}, t_{2}\right)\right) & =d\left(\psi\left(t_{2}\right)\right) & & \text { (if } \left.d \in \Theta^{012}\right) \\
\psi\left(h\left(t_{1}, \cdots, t_{n}\right)\right) & =h\left(\psi\left(t_{1}\right), \cdots, \psi\left(t_{n}\right)\right) & & \text { (otherwise) }
\end{aligned}
$$

Let $R_{2}^{\prime}=R_{1} \cup\{\mathrm{e} \rightarrow 0\} \cup\left\{d(x) \rightarrow d_{2}(0, x) \mid d \in \Theta^{012}\right\}$.

Lemma 4. For any $s \in G_{2}$, if $s \underset{R_{2}^{\prime}}{\longrightarrow} t$ then $\psi(s) \underset{R_{1}}{=} \psi(t)$.

Proof We prove this lemma by induction on the structure of $s$.

Base case: if $s \in Q$ then $s=\psi(s) \underset{R_{1}}{\longrightarrow} \psi(t)=t$.

If $s \in\{\mathrm{e}, 0\}$ then $\psi(s)=\psi(t)=\mathrm{e}$.

\section{Induction step:}

Case of $s \in\left\{\mathrm{f}\left(s_{1}, \cdots, s_{6}\right), \mathrm{g}\left(s_{1}, \cdots, s_{7}\right)\right\}$ : in this case, $\psi(s)=\psi(t)=\mathrm{e}$.

Case of $s=d\left(s_{1}\right)$ where $d \in \Theta^{345}$ : if $t=d\left(t_{1}\right)$ and $s_{1} \underset{R_{2}^{\prime}}{\longrightarrow} t_{1}$ then $\psi(s)=$ $d\left(\psi\left(s_{1}\right)\right) \underset{R_{1}}{=} d\left(\psi\left(t_{1}\right)\right)=\psi(t)$ by the induction hypothesis. Otherwise either $t=d^{\prime}\left(s_{1}\right)$ with $d^{\prime} \in \Theta^{012}$ and $\psi(s)=d\left(\psi\left(s_{1}\right)\right) \underset{R_{1}}{\longrightarrow} d^{\prime}\left(\psi\left(s_{1}\right)\right)=\psi(t)$ or $t=s_{1}$ and $\psi(s)=d\left(\psi\left(s_{1}\right)\right) \underset{R_{1}}{\longrightarrow} \psi\left(s_{1}\right)=\psi(t)$.

Case of $s=d\left(s_{1}\right)$ where $d \in \Theta^{012}$ : if $t=d\left(t_{1}\right)$ and $s_{1} \underset{R_{2}^{\prime}}{\longrightarrow} t_{1}$ then $\psi(s)=$ $d\left(\psi\left(s_{1}\right)\right) \underset{R_{1}}{=} d\left(\psi\left(t_{1}\right)\right)=\psi(t)$ by the induction hypothesis. Otherwise $t=$ $d_{2}\left(0, s_{1}\right)$ and $\psi(s)=\psi(t)=d\left(\psi\left(s_{1}\right)\right)$.

Case of $s=d_{2}\left(s_{1}, s_{2}\right)$ where $d \in \Theta^{012}$ : in this case, $t=d_{2}\left(t_{1}, t_{2}\right)$ holds for some $t_{1}, t_{2}$ and either $s_{1} \underset{R_{2}^{\prime}}{\longrightarrow} t_{1}$ and $s_{2}=t_{2}$ or $s_{2} \underset{R_{2}^{\prime}}{\rightarrow} t_{2}$ and $s_{1}=t_{1}$, hence $\psi(s)=d\left(\psi\left(s_{2}\right)\right) \underset{R_{1}}{=} d\left(\psi\left(t_{2}\right)\right)=\psi(t)$ by the induction hypothesis.

Lemma 5. $0 \underset{R_{2}}{\stackrel{*}{\longrightarrow}} 1$ iff $0 \underset{R_{1}}{\stackrel{*}{\longrightarrow}} 1$.

Proof The if part is obvious. For the only if part, by definition of $R_{2}$, if $0 \underset{R_{2}}{\stackrel{*}{\longrightarrow}} 1$ then there exists a shortest sequence $\gamma$ that satisfies:

$$
\begin{aligned}
\gamma: 0 & \underset{R_{1}}{\longrightarrow} \mathrm{f}\left(q_{A}^{(6)}, q_{A}^{(7)}, q_{A}^{(8)}, q_{A}^{(9)}, q_{B}^{(10)}, q_{B}^{(11)}\right) \stackrel{>\varepsilon *}{R_{2}} \mathrm{f}\left(t_{3}, t_{3}, t_{4}, t_{4}, t_{5}, t_{5}\right) \\
& \underset{R_{1}}{\longrightarrow} \mathrm{g}\left(t_{3}, t_{3}, t_{4}, t_{4}, t_{5}, t_{5}, q_{A}^{(12)}\right) \underset{R_{2}}{\longrightarrow} \mathrm{g}\left(t_{0}, t_{1}, t_{2}, t_{0}, t_{1}, t_{2}, t_{0}\right) \\
& \underset{R_{1}}{\longrightarrow} 1 .
\end{aligned}
$$


Note that $d_{2}(1, x) \rightarrow x$ can not be applied in $\gamma$. Indeed, if $d_{2}(1, x) \rightarrow x$ is applied in $\gamma$, then $\gamma$ must contain a subsequence $0 \underset{R_{2}}{\stackrel{*}{\longrightarrow}} 1$ since 1 appears only in the right-hand side of the rule $\mathrm{g}\left(x_{0}, x_{1}, x_{2}, x_{0}, x_{1}, x_{2}, x_{0}\right) \rightarrow 1, \mathrm{~g}$ is only generated by the rule $\mathrm{f}\left(x_{3}, x_{3}, x_{4}, x_{4}, x_{5}, x_{5}\right) \rightarrow \mathrm{g}\left(x_{3}, x_{3}, x_{4}, x_{4}, x_{5}, x_{5}, q_{A}^{(12)}\right)$, and $\mathrm{f}$ is only generated by the rule $0 \rightarrow \mathrm{f}\left(q_{A}^{(6)}, q_{A}^{(7)}, q_{A}^{(8)}, q_{A}^{(9)}, q_{B}^{(10)}, q_{B}^{(11)}\right)$. This contradicts the hypothesis that $\gamma$ is a shortest sequence. Thus,

$$
\begin{aligned}
& \mathrm{f}\left(q_{A}^{(6)}, q_{A}^{(7)}, q_{A}^{(8)}, q_{A}^{(9)}, q_{B}^{(10)}, q_{B}^{(11)}\right) \stackrel{>\varepsilon *}{R_{2}^{\prime}} \mathrm{f}\left(t_{3}, t_{3}, t_{4}, t_{4}, t_{5}, t_{5}\right) \\
& \text { and } \quad \mathrm{g}\left(t_{3}, t_{3}, t_{4}, t_{4}, t_{5}, t_{5}, q_{A}^{(12)}\right) \frac{>\varepsilon^{\prime} *}{R_{2}^{\prime}} \mathrm{g}\left(t_{0}, t_{1}, t_{2}, t_{0}, t_{1}, t_{2}, t_{0}\right)
\end{aligned}
$$

By Lemma 4 (for sake of readability, we shall write below $\underline{x}$ instead of $\psi(x)$ ):

$$
\begin{aligned}
& \mathrm{f}\left(\underline{q_{A}^{(6)}}, \underline{q_{A}^{(7)}}, \underline{q_{A}^{(8)}}, \underline{q_{A}^{(9)}}, \underline{q_{B}^{(10)}}, \underline{q_{B}^{(11)}}\right) \underset{R_{1}}{\stackrel{*}{(12)}}\left(\underline{t_{3}}, \underline{t_{3}}, \underline{t_{4}}, \underline{t_{4}}, \underline{t_{5}}, \underline{t_{5}}\right) \\
& \text { and } \quad \mathrm{g}\left(\underline{t_{3}}, \underline{t_{3}}, \underline{t_{4}}, \underline{t_{4}}, \underline{t_{5}}, \underline{t_{5}}, \underline{q_{A}^{(12)}}\right) \underset{R_{1}}{\stackrel{*}{\longrightarrow}} \mathrm{g}\left(\underline{t_{0}}, \underline{t_{1}}, \underline{t_{2}}, \underline{t_{0}}, \underline{t_{1}}, \underline{t_{2}}, \underline{t_{0}}\right)
\end{aligned}
$$

Since $\psi(q)=q$ for every $q \in Q, 0 \underset{R_{1}}{\longrightarrow} \mathrm{f}\left(\underline{q_{A}^{(6)}}, \underline{q_{A}^{(7)}}, \underline{q_{A}^{(8)}}, \underline{q_{A}^{(9)}}, \underline{q_{B}^{(10)}}, \underline{q_{B}^{(11)}}\right)$.

By definition of $R_{1}, \mathrm{f}\left(\underline{t_{3}}, \underline{t_{3}}, \underline{t_{4}}, \underline{t_{4}}, \underline{t_{5}}, \underline{t_{5}}\right) \underset{R_{1}}{\longrightarrow} \mathrm{g}\left(\underline{t_{3}}, \underline{t_{3}}, \underline{t_{4}}, \underline{t_{4}}, \underline{t_{5}}, \underline{t_{5}}, \underline{q_{A}^{(12)}}\right)$ and $\mathrm{g}\left(\underline{t_{0}}, \underline{t_{1}}, \underline{t_{2}}, \underline{t_{0}}, \underline{t_{1}}, \underline{t_{2}}, \underline{t_{0}}\right) \underset{R_{1}}{\longrightarrow} 1$. Altogether $0 \underset{R_{1}}{\stackrel{*}{\longrightarrow}} 1$.

We shall show next that $R_{2}$ is confluent iff $0 \underset{R_{2}}{\stackrel{*}{\longrightarrow}} 1$ by using Lemma 3 . We need the following lemma for that purpose.

Lemma 6. If $0 \underset{R_{2}}{\stackrel{*}{\longrightarrow}} 1$ then $t \underset{R_{2}}{\stackrel{*}{\longrightarrow}} 1$ for any $t \in G_{2}$.

Proof First, we note that for any $q \in Q$, there exists $s \in G_{2}$ which does not contain any function symbol in $Q$ such that $q \underset{R_{2}}{\stackrel{*}{R}} s$. Since both of the automata $A$ and $B$ are clean, there exists $u \in \Delta^{(3)^{*}} \cup \Delta^{(4)^{*}} \cup \Sigma^{(5)^{*}} \cup \Delta^{(0)^{*}}$ such that $q \stackrel{*}{\stackrel{*}{R_{0}}} u(\mathrm{e})$.

Thus, it suffices to show that for any $t \in G_{2}$ which does not contain any function symbol in $Q, t \underset{R_{2}}{\stackrel{*}{\longrightarrow}} 1$. We show this proposition by induction on the structure of $t$ :

Base case: by e $\underset{R_{2}}{\longrightarrow} \underset{R_{2}}{\stackrel{*}{\longrightarrow}} 1$.

Induction step: let $t=h\left(t_{1}, \cdots, t_{n}\right)$ where $n>0$ and $h \in \Theta^{012} \cup \Theta^{345} \cup\{\mathrm{f}, \mathrm{g}\} \cup$ $\Theta_{2}^{012}$. By the induction hypothesis, $h\left(t_{1}, \cdots, t_{n}\right) \underset{R_{2}}{\stackrel{*}{\longrightarrow}} h(1, \cdots, 1)$.

For every $d \in \Theta^{345}, d(1) \underset{R_{1}}{\longrightarrow} d^{\prime}(1)$ for some $d^{\prime} \in \Theta^{012}$ or $d(1) \underset{R_{1}}{\longrightarrow}$.

For every $d^{\prime} \in \Theta^{012}, d^{\prime}(1) \underset{R_{2}}{\longrightarrow} d_{2}^{\prime}(0,1) \underset{R_{2}}{\stackrel{*}{\longrightarrow}} d_{2}^{\prime}(1,1)$.

For every $d_{2}^{\prime} \in \Theta_{2}^{012}, d_{2}^{\prime}(1,1) \underset{R_{2}}{\longrightarrow} 1$.

Moreover, $\mathrm{f}(1, \cdots, 1) \underset{R_{1}}{\longrightarrow} \mathrm{g}\left(1, \cdots, 1, q_{A}^{(12)}\right) \underset{\substack{R_{1} \\ R_{2}}}{\stackrel{*}{\longrightarrow}} \mathrm{g}(1, \cdots, 1, u(\mathrm{e}))$

$\underset{R_{1}}{\stackrel{R_{2}}{\longrightarrow}} 1$ where $u \in \Delta^{(0)^{*}}$ 
Let $\phi(t)$ be the term obtained from $t$ by replacing every maximal ground subterm (w.r.t. $\geq_{\text {sub }}$ ) by 1 . Note that:

$$
\begin{aligned}
& \phi\left(R_{0}\right)=P^{(3,0)} \cup \Pi_{1}^{(3,1)} \cup \Pi_{2}^{(4,2)} \cup P^{(4,0)} \cup S^{(5,1)} \cup S^{(5,2)} \\
& \phi\left(R_{1}\right)=\phi\left(R_{0}\right) \cup\left\{\begin{array}{c}
\mathrm{f}\left(x_{3}, x_{3}, x_{4}, x_{4}, x_{5}, x_{5}\right) \rightarrow \mathrm{g}\left(x_{3}, x_{3}, x_{4}, x_{4}, x_{5}, x_{5}, 1\right), \\
\mathrm{g}\left(x_{0}, x_{1}, x_{2}, x_{0}, x_{1}, x_{2}, x_{0}\right) \rightarrow 1
\end{array}\right\} \\
& \phi\left(R_{2}\right)=\phi\left(R_{1}\right) \cup\left\{d(x) \rightarrow d_{2}(1, x), d_{2}(1, x) \rightarrow x \mid d \in \Theta^{012}\right\} .
\end{aligned}
$$

Note also that the rules of $T_{A}$ and $T_{B}$ vanish in $\phi\left(R_{0}\right)$. The following technical lemma is used in the proof of Lemma 8.

Lemma 7. For any non-constant function symbol $h \in \Theta^{012} \cup \Theta^{345} \cup\{\mathrm{f}, \mathrm{g}\} \cup \Theta_{2}^{012}$, $h(1, \cdots, 1) \underset{\phi\left(R_{2}\right)}{\stackrel{*}{\longrightarrow}} 1$.

\section{Proof}

For every $d \in \Theta^{345}, d(1) \underset{\phi\left(R_{1}\right)}{\longrightarrow} d^{\prime}(1)$ for some $d^{\prime} \in \Theta^{012}$ or $d(1) \underset{\phi\left(R_{1}\right)}{\longrightarrow} 1$.

For every $d^{\prime} \in \Theta^{012}, d^{\prime}(1) \underset{\phi\left(R_{2}\right)}{\longrightarrow} d_{2}^{\prime}(1,1)$.

For every $d_{2}^{\prime} \in \Theta_{2}^{012}, d_{2}^{\prime}(1,1) \underset{\phi\left(R_{2}\right)}{\longrightarrow} 1$.

Moreover, $\mathrm{f}(1, \cdots, 1) \underset{\phi\left(R_{1}\right)}{\longrightarrow} \mathrm{g}(1, \cdots, 1) \underset{\phi\left(R_{1}\right)}{\longrightarrow} 1$. Thus, the lemma holds.

We show now how the hypotheses of Lemma 3 hold for $R_{2}$ and $\phi$.

Lemma 8. If $0 \underset{R_{2}}{\stackrel{*}{\longrightarrow}} 1$ then the following properties hold.

(1) If $s \underset{R_{2}}{\longrightarrow} t$ then $\phi(s) \underset{\phi\left(R_{2}\right)}{\longrightarrow} \phi(t)$.

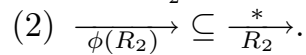

(3) $t \underset{R_{2}}{\stackrel{*}{\longrightarrow}} \phi(t)$.

(4) $\phi\left(R_{2}\right)$ is confluent.

\section{Proof}

(1) By induction on the structure of $s$. If $s$ is a ground term then $\phi(s)=\phi(t)=1$. Thus, we assume that $s$ is not ground. Let $s \frac{p}{R_{2}} t$.

If $p=\varepsilon$ then $s=\alpha \theta \rightarrow \beta \theta=t$ where $\alpha \rightarrow \beta \in R_{2}$. Let $s=h\left(s_{1}, \cdots, s_{n}\right)$ for some $h \in \Theta^{012} \cup \Theta^{345} \cup\{\mathrm{f}, \mathrm{g}\} \cup \Theta_{2}^{012}$ and $s_{1}, \cdots, s_{n}$, and $\alpha=h\left(a_{1}, \cdots, a_{n}\right)$. Since $R_{2}$ is flat, $a_{1} \cdots a_{n} \in X \cup F_{0}$. If $a_{i}$ is a variable then $\phi\left(a_{i} \theta\right)=a_{i} \phi(\theta)$. If $a_{i}$ is a constant then $\phi\left(s_{i}\right)=\phi\left(a_{i}\right)=1$. Thus, $\phi(s)=\phi(\alpha) \phi(\theta)$. Similarly, $\phi(t)=\phi(\beta) \phi(\theta)$, so $\phi(s) \underset{\phi\left(R_{2}\right)}{\longrightarrow} \phi(t)$ holds.

If $p \neq \varepsilon$ then $s=h\left(s_{1}, \cdots, s_{i}, \cdots, s_{n}\right), t=h\left(s_{1}, \cdots, t_{i}, \cdots, s_{n}\right)$, and $s_{i} \underset{R_{2}}{\longrightarrow}$ $t_{i}$ where $i \in\{1, \cdots, n\}$. Since $s$ is not ground,

$$
\phi(s)=h\left(\phi\left(s_{1}\right), \cdots, \phi\left(s_{i}\right), \cdots, \phi\left(s_{n}\right)\right) .
$$

By the induction hypothesis, $\phi\left(s_{i}\right) \underset{\phi\left(R_{2}\right)}{\stackrel{*}{\longrightarrow}} \phi\left(t_{i}\right)$.

If $t$ is not ground then $h\left(\phi\left(s_{1}\right), \cdots, \phi\left(t_{i}\right), \cdots, \phi\left(s_{n}\right)\right)=\phi(t)$.

If $t$ is ground then $h\left(\phi\left(s_{1}\right), \cdots, \phi\left(t_{i}\right), \cdots, \phi\left(s_{n}\right)\right)=h(1, \cdots, 1)$.

By Lemma $7, h(1, \cdots, 1) \underset{\phi\left(R_{2}\right)}{\stackrel{*}{\longrightarrow}} 1=\phi(t)$. Thus, $\phi(s) \underset{\phi\left(R_{2}\right)}{\stackrel{*}{\longrightarrow}} \phi(t)$ holds. 
(2) Since $\phi\left(R_{2}\right) \backslash\left(\left\{d(x) \rightarrow d_{2}(1, x) \mid d \in \Theta^{012}\right\} \cup\left\{\mathrm{f}\left(x_{3}, x_{3}, x_{4}, x_{4}, x_{5}, x_{5}\right) \rightarrow\right.\right.$ $\left.\left.\mathrm{g}\left(x_{3}, x_{3}, x_{4}, x_{4}, x_{5}, x_{5}, 1\right)\right\}\right) \subseteq R_{2}$, it suffices to show that:

$$
\begin{aligned}
d(x \theta) & \stackrel{*}{R_{2}} d_{2}(1, x \theta) \\
\text { and } \mathrm{f}\left(x_{3} \theta, x_{3} \theta, x_{4} \theta, x_{4} \theta, x_{5} \theta, x_{5} \theta\right) & \stackrel{*}{R_{2}} \mathrm{~g}\left(x_{3} \theta, x_{3} \theta, x_{4} \theta, x_{4} \theta, x_{5} \theta, x_{5} \theta, 1\right)
\end{aligned}
$$

We have that: $d(x \theta) \underset{R_{2}}{\longrightarrow} d_{2}(0, x \theta) \underset{R_{2}}{\stackrel{*}{\longrightarrow}} d_{2}(1, x \theta)$.

By Lemma 6:

$$
\begin{aligned}
\mathrm{f}\left(x_{3} \theta, x_{3} \theta, x_{4} \theta, x_{4} \theta, x_{5} \theta, x_{5} \theta\right) & \underset{R_{1}}{\longrightarrow} \mathrm{g}\left(x_{3} \theta, x_{3} \theta, x_{4} \theta, x_{4} \theta, x_{5} \theta, x_{5} \theta, q_{A}^{(12)}\right) \\
\underset{R_{2}}{\longrightarrow} & \mathrm{g}\left(x_{3} \theta, x_{3} \theta, x_{4} \theta, x_{4} \theta, x_{5} \theta, x_{5} \theta, 1\right)
\end{aligned}
$$

(3) By Lemma 6.

(4) We can easily show that $\phi\left(R_{2}\right)$ is terminating by using a lexicographic path order induced by a precedence $>$ that satisfies the following conditions: for any $d \in \Theta^{345}, d^{\prime} \in \Theta^{012}, d^{\prime \prime} \in \Theta_{2}^{012}, d>d^{\prime}>d^{\prime \prime}>1$ and $\mathrm{f}>\mathrm{g}>1$. Thus, it suffices to show that every critical peak of $\phi\left(R_{2}\right)$ is joinable.

For every $a, a^{\prime} \in \Sigma,\left\langle n, a, a^{\prime}\right\rangle^{(0)}(x) \leftarrow\left\langle n, a, a^{\prime}\right\rangle^{(3)}(x) \rightarrow a^{(1)}(x)$ is joinable by: $\left\langle n, a, a^{\prime}\right\rangle^{(0)}(x) \rightarrow\left\langle n, a, a^{\prime}\right\rangle_{2}^{(0)}(1, x) \rightarrow x \leftarrow a_{2}^{(1)}(1, x) \leftarrow a^{(1)}(x)$.

For every $a^{\prime} \in \Sigma,\left\langle n,,_{-}, a^{\prime}\right\rangle^{(0)}(x) \leftarrow\left\langle n,{ }_{-}, a^{\prime}\right\rangle^{(3)}(x) \rightarrow x$ is joinable by: $\left\langle n,,_{-}, a^{\prime}\right\rangle^{(0)}(x) \rightarrow\left\langle n,_{-}, a^{\prime}\right\rangle_{2}^{(0)}(1, x) \rightarrow x$.

For every $a, a^{\prime} \in \Sigma,\left\langle n, a, a^{\prime}\right\rangle^{(0)}(x) \leftarrow\left\langle n, a, a^{\prime}\right\rangle^{(4)}(x) \rightarrow a^{\prime(2)}(x)$ is joinable by: $\left\langle n, a, a^{\prime}\right\rangle^{(0)}(x) \rightarrow\left\langle n, a, a^{\prime}\right\rangle_{2}^{(0)}(1, x) \rightarrow x \leftarrow a_{2}^{\prime(2)}(1, x) \leftarrow a^{\prime(2)}(x)$.

For every $a \in \Sigma,\left\langle n, a,{ }_{-}\right\rangle^{(0)}(x) \leftarrow\left\langle n, a,{ }_{-}\right\rangle^{(4)}(x) \rightarrow x$ is joinable by: $\left\langle n, a,{ }_{-}\right\rangle^{(0)}(x) \rightarrow\left\langle n, a,{ }_{-}\right\rangle_{2}^{(0)}(1, x) \rightarrow x$.

For every $a \in \Sigma, a^{(1)}(x) \leftarrow a^{(5)}(x) \rightarrow a^{(2)}(x)$ is joinable by:

$$
a^{(1)}(x) \rightarrow a_{2}^{(1)}(1, x) \rightarrow x \leftarrow a_{2}^{(2)}(1, x) \leftarrow a^{(2)}(x) .
$$

Lemma 9. $R_{2}$ is confluent iff $0 \stackrel{*}{\stackrel{*}{R_{2}}} 1$.

Proof The if part follows from Lemmata 3 and 8 .

For the only if part, by $\left\langle n,{ }_{-}, a^{\prime}\right\rangle^{(0)}(x) \underset{P^{(3,0)}}{\stackrel{ }{2}}\left\langle n,{ }_{-}, a^{\prime}\right\rangle^{(3)}(x) \underset{\Pi_{1}^{(3,1)}}{\longrightarrow} x$, the confluence ensures that $\left\langle n,{ }_{-}, a^{\prime}\right\rangle^{(0)}(x) \downarrow_{R_{2}} x$.

Since $x$ is a normal form, $\left\langle n,{ }_{-}, a^{\prime}\right\rangle^{(0)}(x) \underset{R_{2}}{\stackrel{*}{\longrightarrow}} x$. Thus, there exists a sequence:

$$
\left\langle n,{ }_{-}, a^{\prime}\right\rangle^{(0)}(x) \underset{R_{2}}{\longrightarrow}\left\langle n,{ }_{-}, a^{\prime}\right\rangle_{2}^{(0)}(0, x) \underset{R_{2}}{\stackrel{*}{\longrightarrow}}\left\langle n,{ }_{-}, a^{\prime}\right\rangle_{2}^{(0)}(1, x) \underset{R_{2}}{\longrightarrow} x
$$

It follows that $0 \underset{R_{2}}{\stackrel{*}{\longrightarrow}} 1$ holds.

By Lemmata 2, 5, 9, the following theorem holds.

Theorem 2. Confluence is undecidable for flat TRSs.

The above TRS $R_{2}$ differs from the analogous one of [8]. Indeed, in some cases, with the TRS of [8] we may have $0 \underset{R_{2}}{\stackrel{*}{\longrightarrow}} 1$ whereas $0 \underset{R_{1}}{\stackrel{*}{\longrightarrow}} 1$ does not hold, which is a problem for the correctness of the reduction. This error was corrected in [9], but Lemma 9 does not hold for the TRS of this report. Therefore, the above TRS $R_{2}$ and the above proof differ from the ones of [9]. 


\section{Concluding remarks}

We have shown that the properties of reachability, joinability and confluence are undecidable for flat TRSs. These results are negative solutions to the open problems posed in [7], and striking compared with the results that the word and unification problems for shallow TRSs are decidable [3]. The undecidability of reachability is shown by a reduction of the Post's Correspondence Problem and the case of joinability and confluence are treated both by a reduction of reachability (with a non trivial reduction in the case of confluence).

The proof techniques involved in our constructions, namely term coloring in Section 3, the criteria for confluence of Lemma 3 and the ground term mapping of Section 4 appeared to be very useful in this context and we believe that they could be of benefit to other decision problems.

Note that the only rules not linear in our TRS are $\mathrm{f}\left(x_{3}, x_{3}, x_{4}, x_{4}, x_{5}, x_{5}\right) \rightarrow$ $\mathrm{g}\left(x_{3}, x_{3}, x_{4}, x_{4}, x_{5}, x_{5}, q_{A}^{(12)}\right)$ and $\mathrm{g}\left(x_{0}, x_{1}, x_{2}, x_{0}, x_{1}, x_{2}, x_{0}\right) \rightarrow 1$ (both left and right members of the first rule are non-linear and they share variables, which is crucial in our reduction). Hence, we have narrowed dramatically the gap between known decidable and undecidable cases of confluence, reachability and joinability of TRS. All three properties are indeed decidable for TRSs whose left members of rules are flat and right members are flat and linear $[17,12,16]$.

It will be a next step to find non-right-linear subclasses of flat (or shallow) TRSs with the decidable property for some of these decision problems. For example, what about the class of flat and semi-constructor TRSs? Here, a semi-constructor TRS is such a TRS that all defined symbols appearing in the right-hand side of each rewrite rule occur only in its ground subterms.

Another interesting question is: does there exist a subclass of TRSs such that exactly one of reachability and confluence is decidable? For the related question about whether there exists a subclass such that exactly one of reachability and joinability is decidable, the existence of such a confluent subclass has been shown in $[10,11]$.

\section{Acknowledgements}

We would like to thank Professor Masahiko Sakai of Nagoya University for his helpful comments. This work was supported in part by Grant-in-Aid for Scientific Research 15500009 from Japan Society for the Promotion of Science.

\section{References}

1. F. Baader and T. Nipkow. Term Rewriting and All That. Cambridge University Press, 1998.

2. H. Comon, G. Godoy, and R. Nieuwenhuis. The confluence of ground term rewrite systems is decidable in polynomial time. In Proc. 42nd Symp. Foundations of Computer Science (FOCS'2001), Las Vegas, NV, USA, Oct. 2001, 2001. 
3. H. Comon, M. Haberstrau, and J.-P. Jouannaud. Syntacticness, cycle-syntacticness and shallow theories. Information and Computation, 111(1):154-191, 1994.

4. M. Dauchet, T. Heuillard, P. Lescanne, and S. Tison. Decidability of the confluence of finite ground term rewrite systems and of other related term rewrite systems. Information and Computation, 88:187-201, 1990.

5. N. Dershowitz and J.-P. Jouannaud. Rewrite systems. In Handbook of Theoretical Computer Science, volume B, pages 243-320. Elsevier Science Publishers B. V., 1990.

6. G. Godoy and A. Tiwari. Confluence of shallow right-linear rewrite systems. In Computer Science Logic, 14th Annual Conf., CSL 2005, pages 541-556. LNCS 3634, 2005.

7. G. Godoy, A. Tiwari, and R. Verma. On the confluence of linear shallow term rewrite systems. In In Symp. on Theoretical Aspects of Computer Science(STACS 2003), pages 85-96. LNCS 2607, 2003.

8. F. Jacquemard. Reachability and confluence are undecidable for flat term rewriting systems. Inf.Process.Lett., 87:265-270, 2003.

9. F. Jacquemard. Erratum to the paper: Reachability and confluence are undecidable for flat term rewriting system. Research Report LSV-05-09, 2005.

10. I. Mitsuhashi, M. Oyamaguchi, Y. Ohta, and T. Yamada. The joinability and related decision problems for semi-constructor TRSs. Trans, IPS Japan, 47(5), 2006. To appear.

11. I. Mitsuhashi, M. Oyamaguchi, and T. Yamada. The reachability and related decision problems for monadic and semi-constructor TRSs. To appear in Inf.Process.Lett.

12. T. Nagaya and Y. Toyama. Decidability for left-linear growing term rewriting systems. In Proc.10th RTA, pages 256-270. LNCS 1631, 1999.

13. M. Oyamaguchi. The Church-Rosser property for ground term-rewriting systems is decidable. Theoretical Computer Science, 49(1):43-79, 1987.

14. M. Oyamaguchi. The reachability and joinability problems for right-ground termrewriting systems. J.Inf.Process., 13(3):347-354, 1990.

15. K. Salomaa. Deterministic tree pushdown automata and monadic tree rewriting systems. J.Comput.Syst.Sci., 37:367-394, 1988.

16. T. Takai, Y. Kaji, and H. Seki. Right-linear finite path overlapping term rewriting systems effectively preserve recognizability. In Proc.11th RTA, pages 246-260. LNCS 1833, 2000.

17. A. Tiwari. Deciding confluence of certain term rewriting systems in polynomial time. In Proc. IEEE Symposium on Logic in Computer Science, pages 447-456. IEEE Society, 2002. 